\title{
Prognostic significance of preoperative plasma fibrinogen levels in primary gastrointestinal stromal tumours: a retrospective cohort study
}

Shibo Song ( $\nabla$ songshibo@bjmu.edu.cn )

Beijing Hospital https://orcid.org/0000-0001-6326-0852

\section{Xianglong Cao}

Beijing Hospital

Hongda Pan

Fudan University Shanghai Cancer Center

Maolin Hu

Beijing Hospital

Qiuxia Yan

Beijing Hospital

Jinghai Song

Beijing Tongren Hospital

Hua Yang

Beijing Hospital

Gang Zhao

Beijing Hospital

Gang Xiao

Beijing Hospital

\section{Research article}

Keywords: Gastrointestinal stromal tumours, Fibrinogen, hypercoagulation, prognosis

Posted Date: October 9th, 2019

DOl: https://doi.org/10.21203/rs.2.15881/v1

License: (c) (i) This work is licensed under a Creative Commons Attribution 4.0 International License.

Read Full License

Version of Record: A version of this preprint was published at International Journal of Clinical Oncology on June 23rd, 2020. See the published version at https://doi.org/10.1007/s10147-020-01689-8. 


\section{Abstract}

Background Improved prediction of prognosis for gastrointestinal stromal tumours (GIST) has become increasingly important since the introduction of small molecule tyrosine kinase inhibitors. Here, we aimed to evaluate the prognostic significance of preoperative plasma fibrinogen (Fib) levels in patients with primary GIST and to analyze their correlations with clinicopathological characteristics.Methods A total of 201 previously untreated patients with primary GIST who had underwent radical surgery at our institution between October 2004 and July 2018 were enrolled. Patient demographics, clinicopathological characteristics, preoperative plasma Fib levels and recurrence-free survival (RFS) were analyzed. The optimal cut-off value for Fib levels was calculated using receiver operating characteristic curve analysis. RFS, the primary endpoint, was calculated by the Kaplan-Meier method and compared by the log-rank test. Univariate and multivariate Cox regression models were calculated.Results Patients in high Fib group had a shorter RFS compared with low Fib group $(P<0.001)$. In multivariate analysis, high preoperative plasma Fib levels were detected as an independent adverse prognostic factor $(P=0.004$, hazard ratio $3.443,95 \%$ confidence interval 1.498-7.916). Furthermore, high preoperative plasma Fib levels also indicated a poor prognosis within the modified National Institutes of Health $(\mathrm{mNIH})$ high-risk subgroup $(P=0.013)$. In addition, preoperative plasma Fib levels were showed a positive correlation with several prognostic factors, and even linearly with tumour size (Spearman correlation coefficient $[r]=$ $0.411, \mathrm{P}<0.001)$.Conclusions High preoperative plasma Fib levels may indicate a poor prognosis in patients with primary GIST. As a cost-effective biomarker, preoperative assessment of plasma Fib levels may help to further risk stratification for patients with $\mathrm{mNIH}$ high-risk GIST and instruct the application of target therapy.

\section{Background}

Gastrointestinal Stromal Tumours (GIST) are rare but the most common mesenchymal neoplasms in the gastrointestinal (GI) tract with an annual incidence of approximately 10 per million population $[1,2]$. The malignant potential of GIST ranges from benign tumours to rapidly progressing sarcomas [3].

The only one well-recognized curative treatment for primary GIST is radical resection [4]. However, recurrence rate remains high after curative resection alone, especially for high-risk patients who have a greater than $50 \%$ chance of recurrence at 2 years [5]. Fortunately, the introduction of small molecule tyrosine kinase inhibitors (TKIs) revolutionized the treatment strategies and greatly improved the prognosis of GIST patients $[4,6]$. The well-recognized indications for TKIs are inoperable or metastatic GIST, and GIST with moderate or high risk of recurrence after resection [4]. However, all the current popularized risk-stratification schemes are only based on tumour-specific factors after resection [7-11]. Therefore, in this era of adjuvant TKIs therapy, it is urgent to find some preoperative factors of prognosis and perfect the current risk-stratification schemes, in order to improve the prediction of prognosis and early instruct the application of target therapy. 
In addition to the tumour-specific factors, hypercoagulation are thought to be associated with development of tumours, such as tumour angiogenesis, invasion, progression, and metastasis [12, 13]. And fibrinogen (Fib) is one of the most significant indicators of coagulation $[12,14]$. In the last few years, high preoperative Fib levels have been found to be associated with poor prognosis in various solid tumours [15-21]. However, to our knowledge, there are few studies on the relationship between Fib with prognosis of patients with GIST, and their results were controversial [22, 23]. Furthermore, no studies have explored whether Fib correlates with prognosis within different recurrent risk subgroups. Hence, there is limited evidence confirming the prognostic ability of Fib in GIST.

In the present study, we analyzed the preoperative plasma Fib levels in 201 patients with primary GIST who had undergone radical surgery, seeking to evaluate their prognostic significance in the overall cohort and subgroups, and further analyze their correlation with clinicopathological prognostic factors.

\section{Methods}

\section{Patients}

We retrospectively reviewed the medical records of 237 consecutive patients with primary GIST who had underwent surgery at Beijing Hospital between October 2004 and July 2018. A total of 201 patients were enrolled in this study. This retrospective study was approved by the institutional review board of Beijing Hospital.

Participants in the present study should meet the following inclusion criteria: 1) postoperative confirmed GIST; 2) R0 resection; 3) available plasma Fib levels data within 7 days before operation; 4) age > 18 years. The exclusion criteria were listed as follows: 1) 6 months or less of follow-up data; 2) preoperative imatinib treatment; 3) coexistent hematological disorders, including splenectomy, thromboembolism, or anticoagulant therapy within 3 months before operation; 4) blood transfusion or inflammatory disorders within 2 weeks before operation; 5) synchronous metastasis or other malignancies; 6) acute or chronic liver disease.

Preoperative operability was assessed by using abdominal/pelvic computed tomography or magnetic resonance imaging. Patients were regularly followed up every 3-6 months for 3-5 years, then annually. The follow-up was conducted by telephone and regular outpatient rechecks. During the follow-up, physical examinations, laboratory tests, imaging and endoscopy were performed. The date of last followup visit, recurrence, or death were recorded. The latest follow-up date was February 15, 2019.

Plasma fibrinogen

Data on preoperative plasma Fib levels were retrospectively obtained from blood coagulation analysis before breakfast within 7 days prior to surgery. Plasma Fib levels were measured by Clauss clotting method using automatic blood coagulation analyzer ACL TOP ${ }^{\text {TM }} 700$ (Instrumentation Laboratory, Werfen Group, America) according to the manufacturer's instruction, with its matching thrombin reagent. 
The diagnosis of GIST were well-recognized by histopathologic examination of resection specimen according to Protocol for the Examination of Specimens From Patients With Gastrointestinal Stromal Tumour [24]. Tumour location, size, rupture, and mitotic index (number of mitoses per 50 high-power fields [HPF]) were recorded. The malignant potential for GIST was based on the modified National Institutes of Health (mNIH) risk classification [7].

Statistical analysis

The optimal cut-off value for preoperative Fib levels was calculated using receiver operating characteristic (ROC) curve analysis. Continuous values were assessed using the student's t-test or the Mann-Whitney U-test, and categorical data were compared by the $\chi^{2}$ test, the Fisher's exact test, the MannWhitney U-test or Kruskal-Wallis test, as appropriate. Spearman's rank correlation coefficients were used to examine associations between two continuous variables. Recurrence-free survival (RFS) was defined as a composite endpoint of local recurrence, distant metastasis, or death-from-any-cause, whatever came first. RFS curves were calculated by the Kaplan-Meier product limit method, then compared using the logrank test. Univariate and multivariate Cox proportional hazard regression models were performed to identify associations with RFS. All tests were two-sided, and $\mathrm{P} \otimes 0.05$ was considered statistically significant. Statistical analyses were performed using SPSS 20.0 software.

\section{Results}

ROC curve analysis

A ROC curve for preoperative plasma Fib levels and the prediction of RFS is shown in Figure 1. The area under the ROC curve of Fib was 0.735 (95\% confidence interval [CI]: 0.623-0.847; P $\otimes 0.001$ ). For all of the GIST patients, the optimal cut-off value of $3.62 \mathrm{~g} / \mathrm{L}$ had the highest sensitivity $(65.4 \%)$ and specificity (80.0\%). Patients were categorized into groups of $\mathrm{H}-\mathrm{Fib}(>3.62 \mathrm{~g} / \mathrm{L})$ and L-Fib $(\leq 3.62 \mathrm{~g} / \mathrm{L})$ according to the cut-off value.

Patient demographics and clinicopathological features

Patient demographics and clinicopathological features are summarized in Table 1. There were 110 males $(54.73 \%)$ and 91 females (45.27\%), with a median age of 61 years (range $26-83$ years) at surgery. The most frequent location was the stomach $(145,72.14 \%)$, followed by the jejunum and ileum $(25,12.44 \%)$, duodenum $(13,6.47 \%)$, colon and rectum $(11,5.47 \%)$, and extra-gastrointestinal sites $(7,3.48 \%)$. The median tumour size was $4.2 \mathrm{~cm}$ (range $0.7-22.0 \mathrm{~cm}$ ), and the majority of patients $(65.17 \%$ ) had a mitotic index of $\leq 5 / 50 \mathrm{HPF}$. All of these 201 patients had no evidence of tumour rupture at the time of surgery. According to the $\mathrm{mNIH}$ risk classification [7], 99 patients (49.25\%) were classified in the very low/low-risk group, 102 patients $(50.75 \%)$ in the moderate/high-risk group. Among the 102 patients, 36 patients (35.29\%) received adjuvant imatinib treatment following surgery. 
Associations between preoperative plasma fibrinogen levels and clinicopathological factors

According to the cut-off value of $3.62 \mathrm{~g} / \mathrm{L}$, patients were divided into two groups: 148 patients were in $\mathrm{H}$ Fib group and 53 patients were in L-Fib group (Table 1). Significantly patients in H-Fib group were more likely to be older $(P=0.005)$ and have a larger tumour size $(P \otimes 0.001)$, a greater mitotic index $(P=0.001)$, a higher $\mathrm{mNIH}$ risk classification $(P \otimes 0.001)$, and a lower plasma albumin (Alb) levels $(P=0.011)$. Other features including sex, cardiovascular diseases (CVDs), diabetes, GI bleeding, anemia, body mass index (BMI), Eastern Cooperative Oncology Group (ECOG) performance status, adjuvant imatinib treatment and tumour location were similarly distributed between both groups. The preoperative plasma Fib levels were

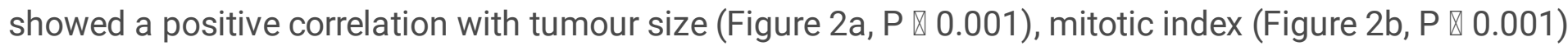
and higher $\mathrm{mNIH}$ risk classification (Figure $2 \mathrm{c}, \mathrm{P} \otimes 0.001$ ), and even linearly with tumour size (Figure 2d, Spearman correlation coefficient $[\lambda]=0.411, \mathrm{P}<0.001)$.

\section{Survival analyses}

The median follow-up time was 45 months(range 7-174 months). Among the 201 patients, 23 patients showed recurrence or metastasis, and 3 patients died from other causes during the study before recurrence or metastasis. For the overall population, the 1-, 3- and 5-year survival rate of RFS was $96.9 \%$, $89.5 \%$ and $84.7 \%$, respectively. Patients in $\mathrm{H}$-Fib group had a shorter RFS (Figure 3, P $\otimes 0.001$ ). The 5-year RFS in H-Fib group was $66.8 \%$, compared to $92.9 \%$ in L-Fib group.

In univariate analysis, Fib (hazard ratio [HR]: 4.576; 95\% Cl: 2.027-10.326; P $\otimes 0.001$ ), tumour location, tumour size and mitotic index were found to be associated with RFS (Table 2). Moreover, multivariate analysis identified Fib (HR: $3.443,95 \% \mathrm{Cl}$ : $1.498-7.916, \mathrm{P}=0.004)$, tumour location and mitotic index as independent predictive factors of RFS (Table 2). High preoperative plasma Fib levels were detected as an independent adverse prognostic factor.

In order to further demonstrate the prognostic significance of Fib, we did subgroup analysis stratified by $\mathrm{mNIH}$ risk, tumour location, adjuvant imatinib treatment and age, respectively. Preoperative plasma Fib levels were still a significantly prognostic factor for GIST within $\mathrm{mNIH}$ high-risk classification (Figure 4a, $\mathrm{P}$ $=0.013$ ) but not for others (Figure $4 \mathrm{~b}, \mathrm{P}=0.088$ ). In addition, high preoperative plasma Fib levels indicated a poor prognosis for GIST regardless of the primary location (Figure $4 \mathrm{c}$ and $4 \mathrm{~d}$ ). In other subgroup analyses, significantly prognostic significance of preoperative plasma Fib levels were showed in patients who did not receive adjuvant imatinib treatment (Figure $4 \mathrm{e}$ and $4 \mathrm{f}$ ) or patients older than 60 years (Figure $4 \mathrm{~g}$ and $4 \mathrm{~h}$ ).

\section{Discussion}

The present study demonstrated that high preoperative plasma Fib levels are significantly associated with poor RFS in patients with primary GIST who underwent radical surgery, and explored its possible cutoff value $(3.62 \mathrm{~g} / \mathrm{L})$ to predict RFS. To the best of our knowledge, This study represents the largest dedicated series published, focusing on the prognostic significance of preoperative plasma Fib levels in 
patients with primary GIST. Furthermore, we found, for the first time, that high preoperative plasma Fib levels still indicated a poor RFS in patients with mNIH high-risk GIST. In addition, our study showed a positive correlation between the preoperative plasma Fib levels and the well-recognized prognostic factors, including tumour size, mitotic index, and $\mathrm{mNIH}$ risk classification. In our study, the correlation between high preoperative plasma Fib levels with poor prognosis was further verified, which is consistent with the previous studies on GIST $[22,23]$. However, the cuff-off value $(3.62 \mathrm{~g} / \mathrm{L})$ is inconsistent with the study by Cai et al (3.24g/L) [23]. Obviously, for the heterogeneity of different populations, it is impossible to determine the ideal threshold for Fib in any small or retrospective study. Although Lu et al first observed the correlation between high preoperative plasma Fib levels with poor prognosis in patients with GIST, their sample size was small $(n=91)$, the follow-up time was shorter (median follow-up time: 2 years) and no any subgroup analysis was performed [22]. Cai et al recently reported a similar result with a larger sample size $(n=170)$, but their data bases overlap with Lu et al and the median follow-up time was not given [22, 23]. In addition, our results are also consistent with previous studies in patients with different malignances [15-21]. Moreover, there were some animal studies suggesting that Fib is an important factor of the metastatic potential of tumour cells $[25,26]$. And some studies have indicated that, in addition to antithrombotic functions, heparins and derivatives also exert critical antimetastatic effects by interference with P-selectin-mediated cell binding [27, 28]. In the present study, tumour size was not observed to be an independent prognostic factor, which may be due to the limited sample size, the exclusion of patients who received preoperative imatinib treatment or non-radical resection, or the obscurity of prognostic significance by other factors. Since the survival analyses were conducted in overall population, the prognostic benefits of adjuvant imatinib treatment were not observed. However, in our study, the adjuvant imatinib treatment significantly improved the prognosis of patients with $\mathrm{mNIH}$ moderate/high-risk GIST ( $P=0.015)$, which is consistent with previous studies $[4,6]$. In the $\mathrm{mNIH}$ high-risk subgroup analysis, for the first time, preoperative plasma Fib levels were still detected to be a significant prognostic factor, which would be conductive to further grading for high-risk patients, and administrating adjuvant therapy to the appropriate patients. However, correlations were not significant within the very low/low/moderate-risk subgroup analysis, which may be due to the limited number of endpoint events and the shorter follow-up time. Preoperative plasma Fib levels were a significant prognostic factor for GIST at both stomach and non-stomach, which may indicate plasma Fib levels were not significantly associated with the tumour locations. For patients without adjuvant imatinib treatment, high preoperative plasma Fib levels indicated a significantly poor prognosis. However, in subgroup with adjuvant imatinib treatment, correlation was not observed between Fib and RFS, which may be due to the prognostic benefits from imatinib treatment or the limited sample size. Our study showed a positive correlation between the preoperative plasma Fib levels and several prognostic factors, including tumour size, mitotic index, and $\mathrm{mNIH}$ risk classification, which is similar to the study by Cai et al [23]. Furthermore, we even observed a linear correlation between preoperative plasma fibrinogen levels and tumour size, which indirectly indicates Fib's prognostic significance. In addition, it was found that patients in H-Fib group had lower plasma concentration of Alb, a biomarker of nutritional status, that was a recognized prognostic factor in many kinds of tumours including GIST [29-31]. Previous studies reported that the plasma concentrations of some coagulation factors, such as Fib, increase progressively with age [32]. We also 
found that patients in $\mathrm{H}$-Fib group were older than patients in L-Fib group, which was in consistent with the previous studies [23,32]. However, for GIST patients, the correlation between age and the preoperative plasma Fib levels was not strong (Spearman correlation coefficient $[r]=0.198, P=0.005$ ). Furthermore, in our study, the high preoperative plasma Fib levels were still significantly associated with the poor RFS in patients older than 60 years. Accordingly, the impact from healthy aging could not cover up the possible correlation between the preoperative plasma Fib levels and the malignant degree of GIST. The reliable molecular mechanisms by which tumour cells interact with the hemostatic system are yet to be clear. Several possible mechanisms were proposed to explain the complex correlation. On the one hand, tumour cells activate the hemostatic system in multiple ways. Tumour cells not only directly activate the coagulation cascade by producing many procoagulant proteins (tissue factor, heparanase, cancer procoagulant, and tissue factor-positive microparticles), but also stimulate the procoagulant properties of the host's hemostatic cells (endothelial cells, platelets, and leukocytes), thereby increasing plasma Fib levels $[33,34]$. We can not also exclude that the pathophysiological mechanism of hypercoagulation may be secondary to tumour-derived systemic inflammatory response and/or intra-abdominal infectious disease [33-36]. Indeed, all of the procoagulant mechanisms elicited by tumour tissues, as well as the patient's general and clinical thrombotic risk factors, contribute to the occurrence of a hypercoagulation of patients with cancer [34]. On the other hand, Fib could also promote tumour progression in return. In tumour microenvironment, Fib could influence the development of tumours through complex interactions with multiple integrin or non-integrin Fib receptors (e.g., cadherins, allb $\beta 3, a V \beta 3, a X \beta 2, a M \beta 2, a 5 \beta 1, a V \beta 1$, Toll-like receptors) which mediate innate immune cell function, tumour cellular proliferation, migration, and apoptosis [37-43]. For example, Fib has been suggested to be a bridging molecule between tumour cells and vascular endothelial growth factor, which could stimulate angiogenesis and promote tumour proliferation [43]. All of these possible mechanisms promote a positive feedback loop between tumour progression and hypercoagulation. As a cost-effective biomarker, the Fib is easily detected from conventional coagulation analysis before surgery. Accordingly, the evaluation of Fib levels would be clinically useful for indicating the malignant potential and prognosis in combination with imaging and pathological features. There are several limitations in the present study. First, a selection bias cannot be excluded due to the nature of a retrospective study in a single institution. The exclusion of patients who did not undergo a radical surgery, as well as the inclusion of patients with adjuvant imatinib treatment, would have an effect on the prognosis of the overall cohort. Second, due to limited follow-up time, the inclusion of patients with adjuvant imatinib treatment and high survival of GIST patients, we did not analyze the OS as the endpoint of this study. Third, genetic mutation analysis was not conducted in most patients because of the high cost, which limits the further studies.

\section{Conclusions}

High preoperative plasma Fib levels may indicate poor prognosis in patients with primary GIST who underwent radical surgery. As a cost-effective biomarker, preoperative assessment of plasma Fib levels may help to further risk stratification for patients with $\mathrm{mNIH}$ high-risk GIST and instruct the application of 
target therapy. However, larger prospective studies and further molecular biological experiments are warranted to confirm our results.

\section{Abbreviations}

GIST: gastrointestinal stromal tumours; Gl: gastrointestinal; TKIs: tyrosine kinase inhibitors; Fib: fibrinogen; HPF: high-power field; mNIH: modified National Institutes of Health; ROC: Receiver operating characteristic; AUC: area under the ROC curve; RFS: recurrence-free survival; $\mathrm{Cl}$ : confidence interval; Alb: albumin; CVDs: cardiovascular diseases; BMI: body mass index; ECOG score: Eastern Cooperative Oncology Group score; HR: hazard ratio

\section{Declarations}

Acknowledgements

The authors are grateful to all medical staff of Department of General Surgery, Beijing Hospital, for their contributions in follow-up and data collection.

Authors' contributions

SBS designed the study, analyzed the data, and wrote the manuscript. GX attended the study design, data interpretation and revision. XLC, HDP, JHS, GZ contributed to study design, and revision. MLH, QXY and HY contributed to the data collection and analysis. All authors read and approved the final manuscript.

Funding

Not applicable.

Availability of data and materials

The datasets used and/or analyzed during the current study are available from the corresponding author on reasonable request.

Ethics approval and consent to participate

The study was approved by the Ethics Committee of Beijing Hospital with the Approval Letter No. 2019BJYYEC-030-01, and was granted an exemption from requiring a informed consent.

Consent for publication

Not applicable.

Competing interests

The authors declare that they have no competing interests. 


\section{References}

1. Miettinen M, Lasota J. Histopathology of gastrointestinal stromal tumor. J Surg Oncol. 2011;104(8):865-873.

2. Gatta G, van der Zwan JM, Casali PG, Siesling S, Dei Tos AP, Kunkler I, Otter R, Licitra L, Mallone S, Tavilla A et al. Rare cancers are not so rare: the rare cancer burden in Europe. Eur $\mathrm{J}$ Cancer. 2011;47(17):2493-2511.

3. von Mehren M, Joensuu H. Gastrointestinal Stromal Tumors. J Clin Oncol. 2018;36(2):136-143.

4. von Mehren M, Randall RL, Benjamin RS, Boles S, Bui MM, Ganjoo KN, George S, Gonzalez RJ, Heslin MJ, Kane JM, 3rd et al. Soft Tissue Sarcoma, Version 2.2018, NCCN Clinical Practice Guidelines in Oncology. J Natl Compr Canc Netw. 2018;16(5):536-563.

5. Dematteo RP, Ballman KV, Antonescu CR, Maki RG, Pisters PW, Demetri GD, Blackstein ME, Blanke CD, von Mehren M, Brennan MF et al. Adjuvant imatinib mesylate after resection of localised, primary gastrointestinal stromal tumour: a randomised, double-blind, placebo-controlled trial. Lancet. 2009;373(9669):1097-1104.

6. Casali PG, Zalcberg J, Le Cesne A, Reichardt P, Blay JY, Lindner LH, Judson IR, Schoffski P, Leyvraz S, Italiano A et al. Ten-Year Progression-Free and Overall Survival in Patients With Unresectable or Metastatic GI Stromal Tumors: Long-Term Analysis of the European Organisation for Research and Treatment of Cancer, Italian Sarcoma Group, and Australasian Gastrointestinal Trials Group Intergroup Phase III Randomized Trial on Imatinib at Two Dose Levels. J Clin Oncol. 2017;35(15):1713-1720.

7. Joensuu $\mathrm{H}$. Risk stratification of patients diagnosed with gastrointestinal stromal tumor. Hum Pathol. 2008;39(10):1411-1419.

8. Miettinen M, Lasota J. Gastrointestinal stromal tumors: pathology and prognosis at different sites. Semin Diagn Pathol. 2006;23(2):70-83.

9. Fletcher CD, Berman JJ, Corless C, Gorstein F, Lasota J, Longley BJ, Miettinen M, O'Leary TJ, Remotti $\mathrm{H}$, Rubin BP et al. Diagnosis of gastrointestinal stromal tumors: A consensus approach. Hum Pathol. 2002;33(5):459-465.

10. Gold JS, Gonen M, Gutierrez A, Broto JM, Garcia-del-Muro X, Smyrk TC, Maki RG, Singer S, Brennan $\mathrm{MF}$, Antonescu CR et al. Development and validation of a prognostic nomogram for recurrence-free survival after complete surgical resection of localised primary gastrointestinal stromal tumour: a retrospective analysis. Lancet Oncol. 2009;10(11):1045-1052.

11. Joensuu H, Vehtari A, Riihimaki J, Nishida T, Steigen SE, Brabec P, Plank L, Nilsson B, Cirilli C, Braconi $\mathrm{C}$ et al. Risk of recurrence of gastrointestinal stromal tumour after surgery: an analysis of pooled population-based cohorts. Lancet Oncol. 2012;13(3):265-274.

12. Repetto $\mathrm{O}$, De Re V. Coagulation and fibrinolysis in gastric cancer. Annals of the New York Academy of Sciences. 2017;1404(1):27-48. 
13. Palumbo JS. Mechanisms linking tumor cell-associated procoagulant function to tumor dissemination. Seminars in thrombosis and hemostasis. 2008;34(2):154-160.

14. Tennent GA, Brennan SO, Stangou AJ, O'Grady J, Hawkins PN, Pepys MB. Human plasma fibrinogen is synthesized in the liver. Blood. 2007;109(5):1971-1974.

15. Huang G, Jiang H, Lin Y, Wu Y, Cai W, Shi B, Luo Y, Jian Z, Zhou X. Prognostic value of plasma fibrinogen in hepatocellular carcinoma: a meta-analysis. Cancer management and research. 2018;10:5027-5041.

16. Matsuda S, Takeuchi H, Kawakubo H, Fukuda K, Nakamura R, Takahashi T, Wada N, Saikawa Y, Omori T, Kitagawa Y. Cumulative prognostic scores based on plasma fibrinogen and serum albumin levels in esophageal cancer patients treated with transthoracic esophagectomy: comparison with the Glasgow prognostic score. Ann Surg Oncol. 2015;22(1):302-310.

17. Yu X, Hu F, Yao Q, Li C, Zhang H, Xue Y. Serum fibrinogen levels are positively correlated with advanced tumor stage and poor survival in patients with gastric cancer undergoing gastrectomy: a large cohort retrospective study. BMC Cancer. 2016;16:480.

18. Lee JH, Hyun JH, Kim DY, Yoo BC, Park JW, Kim SY, Chang HJ, Kim BC, Kim TH, Oh JH et al. The role of fibrinogen as a predictor in preoperative chemoradiation for rectal cancer. Ann Surg Oncol. 2015;22(1):209-215.

19. Qi Q, Geng Y, Sun M, Chen H, Wang P, Chen Z. Hyperfibrinogen Is Associated With the Systemic Inflammatory Response and Predicts Poor Prognosis in Advanced Pancreatic Cancer. Pancreas. 2015;44(6):977-982.

20. Thurner EM, Krenn-Pilko S, Langsenlehner U, Stojakovic T, Pichler M, Gerger A, Kapp KS, Langsenlehner T. The association of an elevated plasma fibrinogen level with cancer-specific and overall survival in prostate cancer patients. World journal of urology. 2015;33(10):1467-1473.

21. Krenn-Pilko S, Langsenlehner U, Stojakovic T, Pichler M, Gerger A, Kapp KS, Langsenlehner T. An elevated preoperative plasma fibrinogen level is associated with poor disease-specific and overall survival in breast cancer patients. Breast (Edinburgh, Scotland). 2015;24(5):667-672.

22. Lu J, Chen S, Li X, Qiu G, He S, Wang H, Zhou L, Jing Y, Che X, Fan L. Gastrointestinal stromal tumors: Fibrinogen levels are associated with prognosis of patients as blood-based biomarker. Medicine (Baltimore). 2018;97(17):e0568.

23. Cai HX, Li XQ, Wang SF. Prognostic value of fibrinogen and D-dimer-fibrinogen ratio in resectable gastrointestinal stromal tumors. World J Gastroenterol. 2018;24(44):5046-5056.

24. Rubin BP, Blanke CD, Demetri GD, Dematteo RP, Fletcher CD, Goldblum JR, Lasota J, Lazar A, Maki $\mathrm{RG}$, Miettinen $\mathrm{M}$ et al. Protocol for the examination of specimens from patients with gastrointestinal stromal tumor. Arch Pathol Lab Med. 2010;134(2):165-170.

25. Palumbo JS, Kombrinck KW, Drew AF, Grimes TS, Kiser JH, Degen JL, Bugge TH. Fibrinogen is an important determinant of the metastatic potential of circulating tumor cells. Blood. 2000;96(10):3302-3309. 
26. Palumbo JS, Potter JM, Kaplan LS, Talmage K, Jackson DG, Degen JL. Spontaneous hematogenous and lymphatic metastasis, but not primary tumor growth or angiogenesis, is diminished in fibrinogendeficient mice. Cancer Res. 2002;62(23):6966-6972.

27. Ludwig RJ, Alban S, Bistrian R, Boehncke WH, Kaufmann R, Henschler R, Gille J. The ability of different forms of heparins to suppress P-selectin function in vitro correlates to their inhibitory capacity on bloodborne metastasis in vivo. Thrombosis and haemostasis. 2006;95(3):535-540.

28. Stevenson JL, Choi SH, Varki A. Differential metastasis inhibition by clinically relevant levels of heparins--correlation with selectin inhibition, not antithrombotic activity. Clin Cancer Res. 2005;11(19 Pt 1):7003-7011.

29. Blanke CD, Rankin C, Demetri GD, Ryan CW, von Mehren M, Benjamin RS, Raymond AK, Bramwell VH, Baker LH, Maki RG et al. Phase III randomized, intergroup trial assessing imatinib mesylate at two dose levels in patients with unresectable or metastatic gastrointestinal stromal tumors expressing the kit receptor tyrosine kinase: S0033. J Clin Oncol. 2008;26(4):626-632.

30. Roxburgh CS, Salmond JM, Horgan PG, Oien KA, McMillan DC. Comparison of the prognostic value of inflammation-based pathologic and biochemical criteria in patients undergoing potentially curative resection for colorectal cancer. Ann Surg. 2009;249(5):788-793.

31. Proctor MJ, Morrison DS, Talwar D, Balmer SM, Fletcher CD, O'Reilly DS, Foulis AK, Horgan PG, McMillan DC. A comparison of inflammation-based prognostic scores in patients with cancer. A Glasgow Inflammation Outcome Study. Eur J Cancer. 2011;47(17):2633-2641.

32. Gligorijevic N, Zamorova Krizakova M, Penezic A, Katrlik J, Nedic O. Structural and functional changes of fibrinogen due to aging. International journal of biological macromolecules. 2018;108:1028-1034.

33. Falanga A, Marchetti M, Vignoli A. Coagulation and cancer: biological and clinical aspects. Journal of thrombosis and haemostasis: JTH. 2013;11(2):223-233.

34. Falanga A, Schieppati F, Russo D. Cancer Tissue Procoagulant Mechanisms and the Hypercoagulable State of Patients with Cancer. Seminars in thrombosis and hemostasis. 2015;41(7):756-764.

35. Racz JM, Cleghorn MC, Jimenez MC, Atenafu EG, Jackson TD, Okrainec A, Venkat Raghavan L, Quereshy FA. Predictive Ability of Blood Neutrophil-to-Lymphocyte and Platelet-to-Lymphocyte Ratios in Gastrointestinal Stromal Tumors. Ann Surg Oncol. 2015;22(7):2343-2350.

36. Zhao L, Feng S, Huang S, Tong Y, Chen Z, Wu P, Lai XH, Chen X. Diagnostic value of hyperfibrinogenemia as a predictive factor for appendiceal perforation in acute appendicitis. ANZ J Surg. 2017;87(5):372-375.

37. Degen JL, Palumbo JS. Mechanisms linking hemostatic factors to tumor growth in mice. Pathophysiology of haemostasis and thrombosis. 2003;33 Suppl 1:31-35.

38. Staton CA, Brown NJ, Lewis CE. The role of fibrinogen and related fragments in tumour angiogenesis and metastasis. Expert opinion on biological therapy. 2003;3(7):1105-1120. 
39. Neufert C, Becker C, Neurath MF. An inducible mouse model of colon carcinogenesis for the analysis of sporadic and inflammation-driven tumor progression. Nature protocols. 2007;2(8):1998-2004.

40. Steinbrecher KA, Harmel-Laws E, Sitcheran R, Baldwin AS. Loss of epithelial RelA results in deregulated intestinal proliferative/apoptotic homeostasis and susceptibility to inflammation. Journal of immunology (Baltimore, Md : 1950). 2008;180(4):2588-2599.

41. Palumbo JS, Talmage KE, Liu H, La Jeunesse CM, Witte DP, Degen JL. Plasminogen supports tumor growth through a fibrinogen-dependent mechanism linked to vascular patency. Blood. 2003;102(8):2819-2827.

42. Steinbrecher KA, Horowitz NA, Blevins EA, Barney KA, Shaw MA, Harmel-Laws E, Finkelman FD, Flick MJ, Pinkerton MD, Talmage KE et al. Colitis-associated cancer is dependent on the interplay between the hemostatic and inflammatory systems and supported by integrin alpha(M)beta(2) engagement of fibrinogen. Cancer Res. 2010;70(7):2634-2643.

43. Simpson-Haidaris PJ, Rybarczyk B. Tumors and fibrinogen. The role of fibrinogen as an extracellular matrix protein. Annals of the New York Academy of Sciences. 2001;936:406-425.

\section{Tables}

Table 1 Patient demographics and clinicopathological features 


\begin{tabular}{|c|c|c|c|c|}
\hline Variable & Total $(n=201)$ & $\begin{array}{l}\text { L-Fib }(n=148) \\
\text { Fib } \leq 3.62 \mathrm{~g} / \mathrm{L}\end{array}$ & $\begin{array}{l}\text { H-Fib }(n=53) \\
\text { Fib }>3.62 \mathrm{~g} / \mathrm{L}\end{array}$ & P-value \\
\hline Age (years)* & $61(52,70)$ & $60(50,68)$ & $64(57,72)$ & $0.005^{a}$ \\
\hline Gender & & & & \\
\hline Male & 110 (54.73\%) & 77 (52.03\%) & 33 (62.26\%) & $0.199^{b}$ \\
\hline Female & 91 (45.27\%) & 71 (47.97\%) & $20(37.74 \%)$ & \\
\hline CVDs & & & & \\
\hline No & 111 (55.22\%) & 82 (55.41\%) & 29 (54.72\%) & $0.931^{\mathrm{b}}$ \\
\hline Yes & $90(44.78 \%)$ & 66 (44.59\%) & 24 (45.28\%) & \\
\hline Diabetes & & & & \\
\hline No & 175 (87.06\%) & 132 (89.19\%) & 43 (81.13\%) & $0.134^{\mathrm{b}}$ \\
\hline Yes & 26 (12.94\%) & 16 (10.81\%) & $10(18.87 \%)$ & \\
\hline GI bleeding & & & & \\
\hline No & 160 (79.60\%) & 121 (81.76\%) & 39 (73.58\%) & $0.205^{\mathrm{b}}$ \\
\hline Yes & 41 (20.40\%) & 27 (18.24\%) & $14(26.42 \%)$ & \\
\hline Anemia & & & & \\
\hline No & 124 (61.69\%) & 96 (64.86\%) & 28 (52.83\%) & $0.122^{\mathrm{b}}$ \\
\hline Yes & 77 (38.31\%) & 52 (35.14\%) & 25 (47.17\%) & \\
\hline BMI $\left(\mathrm{kg} / \mathrm{m}^{2}\right)^{*}$ & $24.22 \pm 3.38$ & $24.33 \pm 3.63$ & $23.90 \pm 2.59$ & $0.351^{\mathrm{C}}$ \\
\hline Alb $(\mathrm{g} / \mathrm{L}) *$ & $40(38,42)$ & $41(39,43)$ & $40(36,41)$ & $0.011^{a}$ \\
\hline ECOG score & & & & \\
\hline $1-2$ & 196 (97.51\%) & 114 (97.30\%) & 52 (98.11\%) & $1.000^{\mathrm{d}}$ \\
\hline $3-4$ & $5(2.49 \%)$ & $4(2.70 \%)$ & $1(1.89 \%)$ & \\
\hline Tumour location & & & 36 (67 $070 /$ & $0125 \mathrm{~b}$ \\
\hline $\begin{array}{l}\text { stomach } \\
\text { Non-stomach }\end{array}$ & $\begin{array}{l}145(/ 2.14 \%) \\
56(27.86 \%)\end{array}$ & $109(13.65 \%)$ & $\begin{array}{l}36(67.92 \%) \\
17(3208 \%)\end{array}$ & $0.425^{\circ}$ \\
\hline Tumour size $(\mathrm{cm})$ & $50(21.0070)$ & (20.0570) & $1 /(32.00 \% 0)$ & \\
\hline$\leq 2$ & 37 (18.41\%) & 34 (22.97\%) & $3(5.66 \%)$ & $0.001^{a}$ \\
\hline$\leq 5$ & $90(44.78 \%)$ & 71 (47.97\%) & 19 (35.85\%) & \\
\hline$\leq 10$ & 44 (21.89\%) & 31 (20.95\%) & 13 (24.53\%) & \\
\hline$>10$ & $30(14.93 \%)$ & $12(8.11 \%)$ & 18 (33.96\%) & \\
\hline Mitotic index (/50HFP) & & & & \\
\hline$\leq 5$ & $131(65.17 \%)$ & 105 (70.95\%) & $26(49.06 \%)$ & $0.001^{a}$ \\
\hline$\leq 10$ & $32(15.92 \%)$ & $24(16.22 \%)$ & $8(15.09 \%)$ & \\
\hline$>10$ & $38(18.91 \%)$ & 19 (12.84\%) & 19 (35.85\%) & \\
\hline mNIH risk classification & & & & \\
\hline Very low & $36(17.91 \%)$ & $33(22.30 \%)$ & $3(5.66 \%)$ & $0.001^{a}$ \\
\hline Low & $63(31.34 \%)$ & $51(34.46 \%)$ & 12 (22.64\%) & \\
\hline Moderate & 37 (18.41\%) & 26 (17.57\%) & 11 (20.76\%) & \\
\hline High & $65(32.34 \%)$ & $38(25.67 \%)$ & 27 (50.94\%) & \\
\hline Postoperative imatinib & & & & \\
\hline No & 165 (82.09\%) & 125 (84.46\%) & 40 (75.47\%) & $0.143^{b}$ \\
\hline Yes & $36(17.91 \%)$ & $23(15.54 \%)$ & $13(24.53 \%)$ & \\
\hline
\end{tabular}

Notes: Continuous variables were expressed as means \pm standard deviations or medians (25th-75th percentile), and categorical variables were presented as frequency (\%), as appropriate. Continuous values were assessed using the student's t-test or the Mann-Whitney U-test, and categorical data were compared by the $\chi^{2}$ test, the Fisher's exact test, or the Mann-Whitney U-test, as appropriate. * Continuous value, a the Mann-Whitney U-test, ${ }^{b}$ the $\mathrm{c}^{2}$ test, $^{\mathrm{c}}$ the student's t-test, ${ }^{\mathrm{d}}$ the Fisher's exact test.

Abbreviations: Fib, fibrinogen; CVDs, cardiovascular diseases; GI, gastrointestinal; BMI, body mass index; Alb, albumin; ECOG score, Eastern Cooperative Oncology Group score; mNIH risk classification, modified National Institutes of Health risk classification. 
Table 2 Univariate and multivariate Cox regression analyses for RFS ( $\mathrm{n}=201)$

\begin{tabular}{|c|c|c|c|c|c|c|}
\hline \multirow[t]{2}{*}{ iable } & \multicolumn{3}{|c|}{ Univariate analysis } & \multicolumn{3}{|c|}{ Multivariate analysis } \\
\hline & HR & $95 \%$ CI & P-value & HR & $95 \%$ CI & P-value \\
\hline$(>3.62 \mathrm{vs} \leq 3.62 \mathrm{~g} / \mathrm{L})$ & 4.576 & $2.027-10.326$ & 0.001 & 3.443 & $1.498-7.916$ & 0.004 \\
\hline : (> 60 vs $\leq 60$ years $)$ & 1.174 & $0.542-2.544$ & 0.684 & & & \\
\hline ıder (male vs female) & 0.842 & $0.381-1.859$ & 0.670 & & & \\
\hline ation (non-stomach vs stomach) & 4.088 & $1.884-8.871$ & प 0.001 & 4.042 & $1.811-9.024$ & 0.001 \\
\hline nour size (>5 vs $\leq 5 \mathrm{~cm})$ & 5.363 & $2.251-12.785$ & 0.001 & & & \\
\hline otic index (> 5 vs $\leq 5 / 50 \mathrm{HPF}$ ) & 8.501 & $3.360-21.507$ & 0.001 & 6.146 & 2.373-15.918 & प 0.001 \\
\hline itoperative imatinib (yes vs no) & 0.593 & $0.177-1.982$ & 0.396 & & & \\
\hline
\end{tabular}

Notes: Univariate and multivariate Cox proportional hazard regression models were performed to identify associations with RFS. Factors found significant in univariate analysis were included in a forward stepwise multivariate Cox proportional hazards regression model with entry criteria of $\mathrm{P} \square 0.05$ and removal criteria of $\mathrm{P}>$ 0.1 .

Abbreviations: Fib, fibrinogen; RFS, recurrence-free survival; HR, hazard ratio; CI, confidence interval; HPF, high-power field.

Figures 


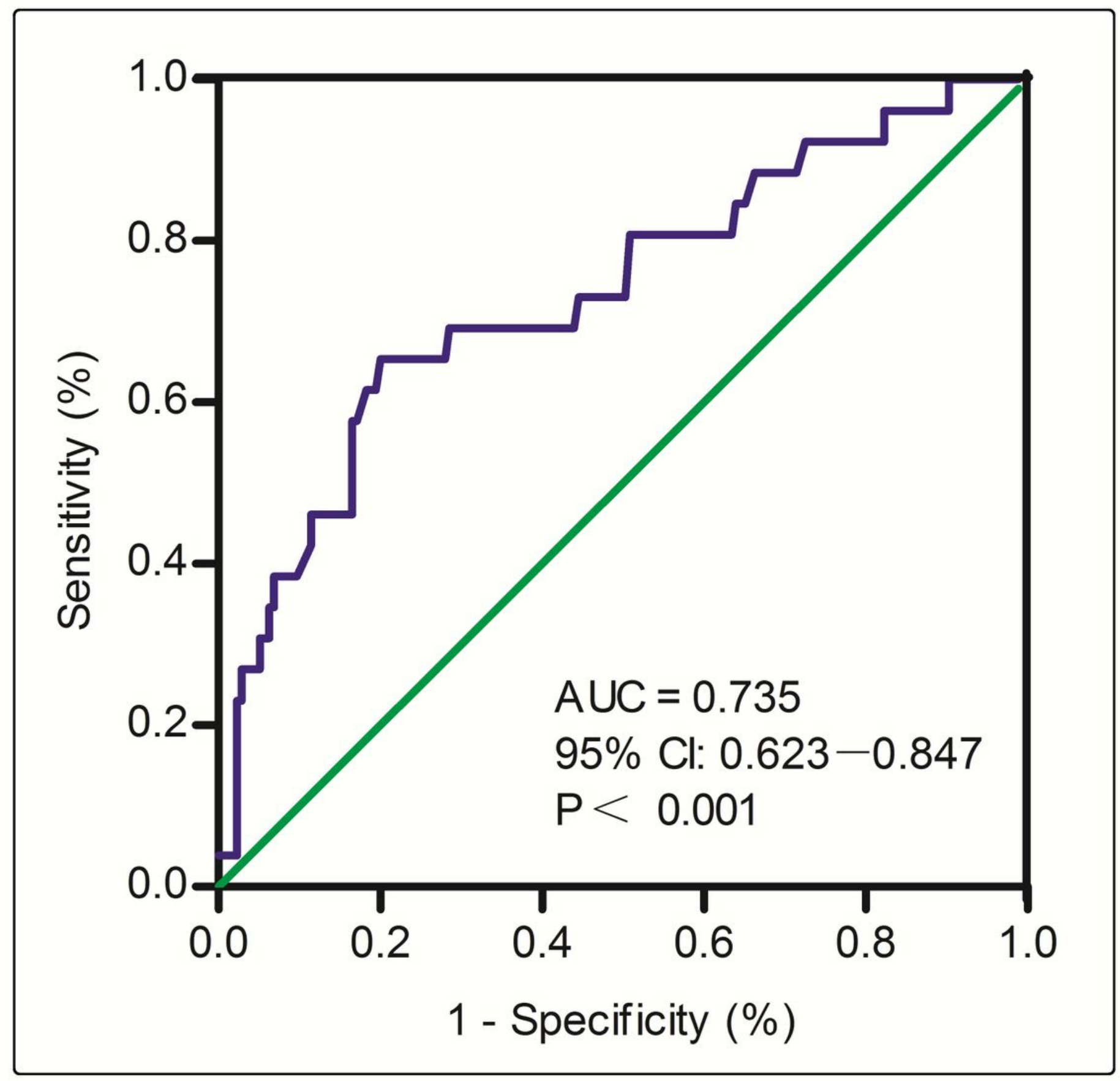

Figure 1

ROC curve for preoperative plasma Fib levels to predict RFS. Notes: The area under the ROC curve of Fib was 0.735 . Fib $=3.62 \mathrm{~g} / \mathrm{L}$ had the highest sensitivity $(65.4 \%)$ and specificity $(80.0 \%)$. The green line represents the reference line, and the blue line represents Fib. Abbreviations: ROC, Receiver operating characteristic; Fib, fibrinogen; RFS, recurrence-free survival; AUC, area under the ROC curve; $\mathrm{Cl}$, confidence interval. 


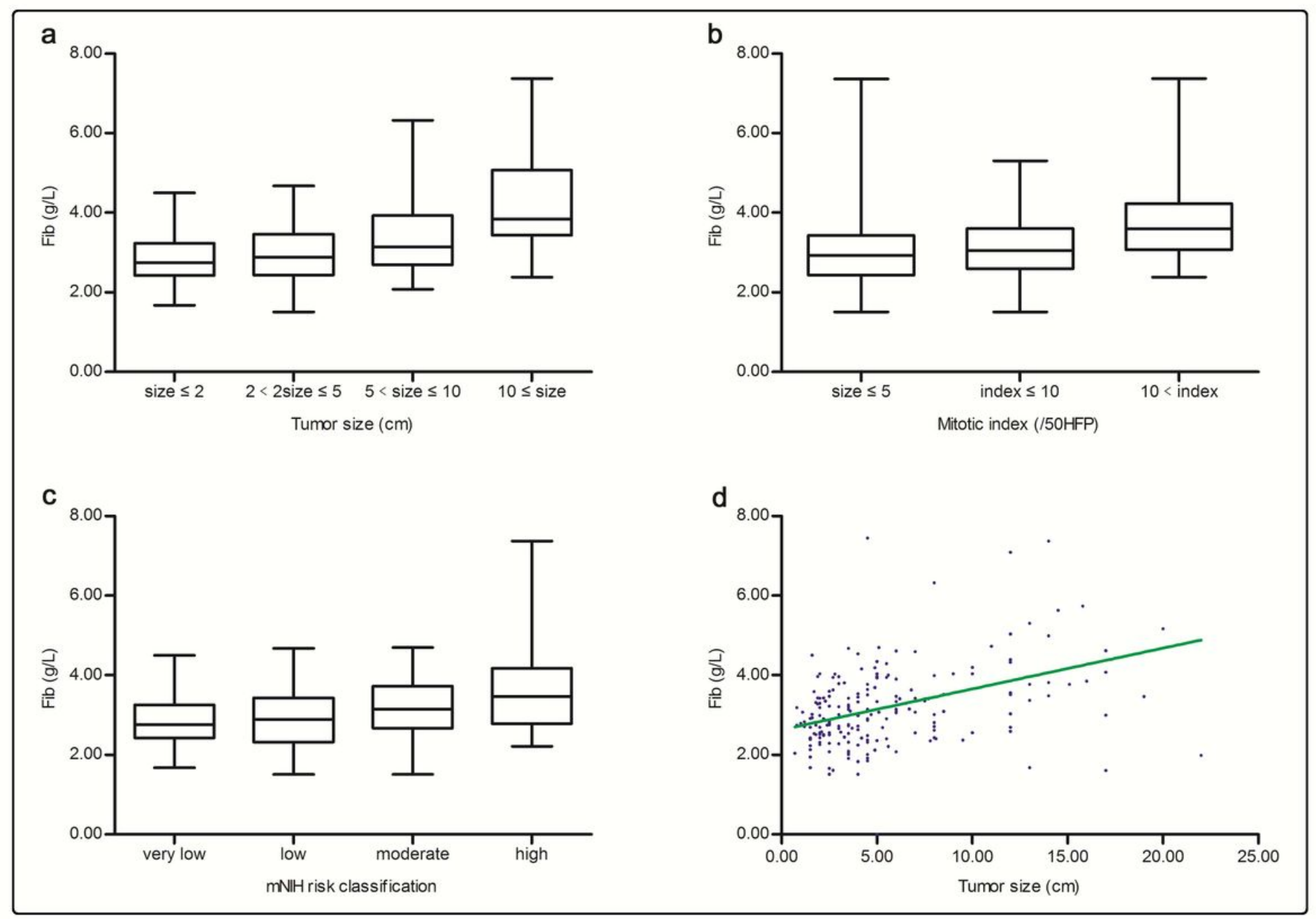

Figure 2

Associations between preoperative plasma Fib levels and several prognostic factors Notes: (a) Preoperative Fib levels in GIST patients in terms of primary tumour size. Kruskal-Wallis test: $P \otimes 0.001$. (b) Preoperative Fib levels in GIST patients in terms of mitotic index. Kruskal-Wallis test: $P \otimes 0.001$. (c) Preoperative Fib levels in GIST patients in terms of the modified National Institutes of Health $(\mathrm{mNIH})$ risk classification. Kruskal-Wallis test: $P \otimes 0.001$. (d) Tumour size is positively associated with preoperative Fib levels (Spearman correlation coefficient $[r]=0.411, P \otimes 0.001$ ). Abbreviations: Fib, fibrinogen; GIST, gastrointestinal stromal tumours; HPF, high-power field; $\mathrm{mNIH}$ risk classification, modified National Institutes of Health risk classification. 


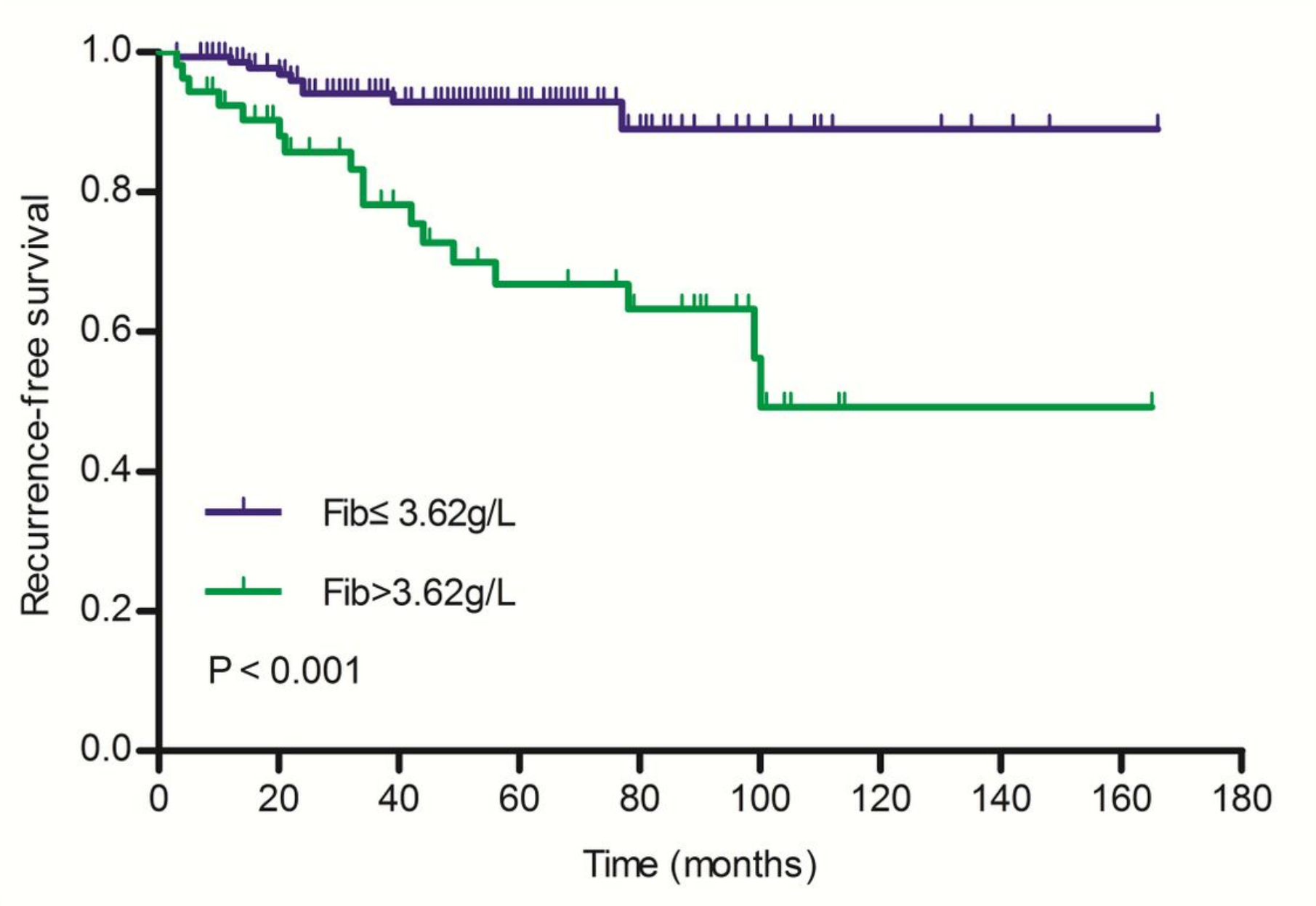

Figure 3

RFS curve analysis for GIST patients based on the preoperative plasma Fib level. Notes: Kaplan-Meier estimate of the censoring time distribution in 201 patients with primary GIST. High preoperative plasma Fib level was significantly associated with shorter RFS. The P-value was calculated by using the log-rank test. Abbreviations: RFS, recurrence-free survival; GIST, gastrointestinal stromal tumour; Fib, fibrinogen. 


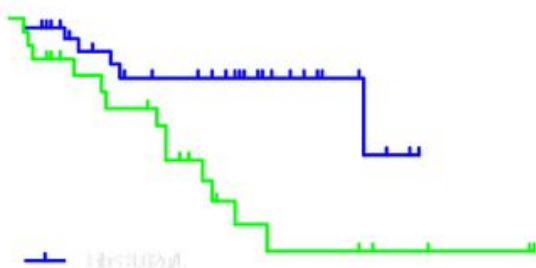

$\pm$

c

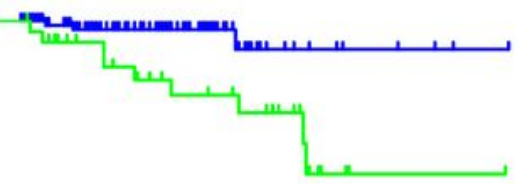

$+$

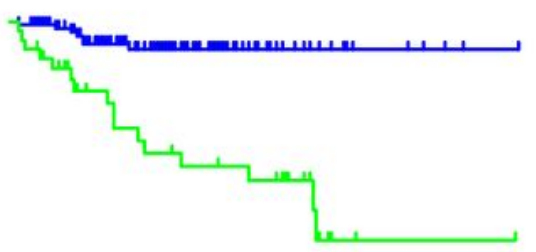

$+$

g

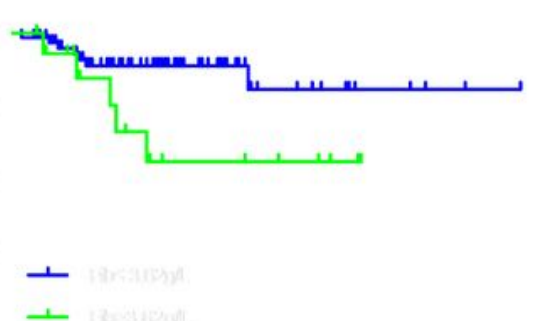

b

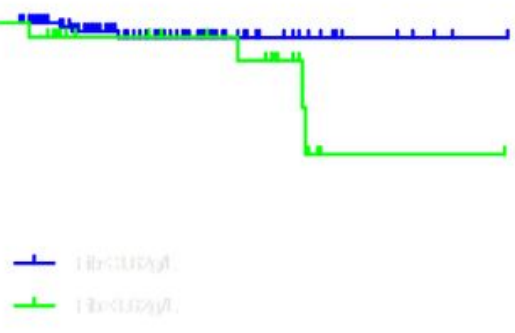

d

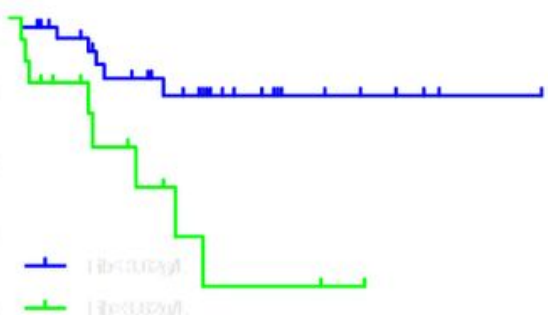

f

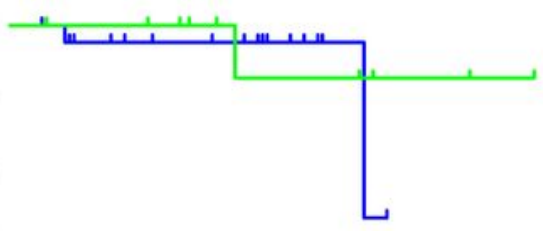

$+$

$+$

h

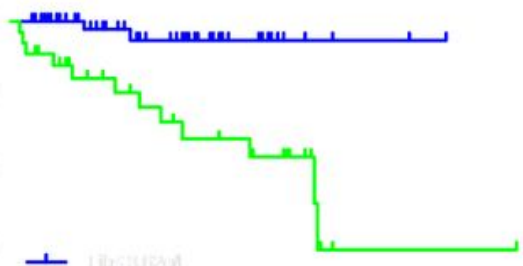

\section{Figure 4}

RFS curve analyses based on the preoperative plasma Fib level in subgroups. Notes: (a) 65 patients with $\mathrm{mNIH}$ high-risk GIST ( $\mathrm{P}=0.013)$, (b) 112 patients with $\mathrm{mNIH}$ very low/low/moderate-risk GIST $(P=0.088)$ (c) 123 patients with GIST at stomach $(P=0.004)$, (d) 43 patients with GIST at non-stomach $(P=0.005)$, (e) 133 patients without adjuvant imatinib treatment $(P \otimes 0.001)$, (f) 33 patients with adjuvant imatinib treatment $(P=0.717),(\mathrm{g}) 80$ patients $\leq 60(P=0.052)$, $(\mathrm{h}) 86$ patients $>60$ years $(P=0.001)$. 
Abbreviations: RFS, recurrence-free survival; Fib, fibrinogen; $\mathrm{mNIH}$ risk classification, modified National Institutes of Health risk classification; GIST, gastrointestinal stromal tumour. 